Copyright ( 2013 IEEE. Personal use of this material is permitted. Permission from IEEE must be obtained for all other uses, in any current or future media, including reprinting/republishing this material for advertising or promotional purposes, creating new collective works, for resale or redistribution to servers or lists, or reuse of any copyrighted component of this work in other works. 


\title{
Iterative Frequency Domain Equalization for Generalized Approximate Message Passing
}

\author{
Qinghua Guo, Defeng (David) Huang, Sven Nordholm, Jiangtao Xi, and Yanguang Yu
}

\begin{abstract}
An iterative frequency domain equalization approach for coded single-carrier block transmissions over frequency selective channels is developed by using the recently proposed generalized approximate message passing (GAMP) algorithm. Compared with the low-complexity iterative frequency domain linear minimum mean square error (FD-LMMSE) equalization approach, the proposed one can achieve significant performance gain with slight complexity increase.
\end{abstract}

Index Terms-Turbo equalization, frequency domain equalization, linear MMSE, approximate message passing, generalized approximate message passing.

\section{INTRODUCTION}

$\mathbf{T}$ URBO (iterative) equalization is a powerful technique to combat the inter-symbol interference (ISI) and it can achieve impressive performance gain through the iterative extrinsic information exchange between a soft-in soft-out (SISO) decoder and a SISO equalizer as shown in Fig. 1 [1]-[12]. Since the conception of turbo equalization in 1995, it has received tremendous attention with a focus on the implementation of the SISO equalizer [2]-[12].

The original APP (a posteriori probability)-based SISO equalizer provides optimal performance but at the cost of prohibitive computational complexity [1], which grows exponentially with the number of channel taps $L$. This motivated the invention of the linear MMSE (minimum mean square error) SISO equalizer with complexity reduced to $\mathcal{O}\left(L^{2}\right)$ [2][7]. However, the complexity is still a concern when the number of channel taps $L$ is large, e.g., in broadband wireless communications and underwater acoustic communications, $L$ can be tens even a few hundreds [13], [14]. Recently, the linear MMSE equalizer has been implemented in the frequency domain (with or without the assist of cyclic prefixing), reducing complexity to the logarithmic level [8], [10]-[12]. The frequency domain linear MMSE (FD-LMMSE) equalizer is an attractive option due to its low complexity. However, it may suffer from significant performance loss when the transmitted signal is severely distorted by an ISI channel.

Q. Guo is with the School of Electrical, Computer and Telecommunications Engineering, University of Wollongong, NSW, Australia, and the School of Electrical, Electronic and Computer Engineering, the University of Western Australia, WA, Australia (e-mail: qinghua_guo@uow.edu.au).

D. Huang is with the School of Electrical, Electronic and Computer Engineering, the University of Western Australia, WA, Australia (e-mail: david.huang@uwa.edu.au).

S. Nordholm is with the Department of Electrical and Computer Engineering, Curtin University, WA, Australia (email: s.nordholm@ curtin.edu.au).

J. Xi and Y. Yu are with the School of Electrical, Computer and Telecommunications Engineering, University of Wollongong, NSW, Australia (e-mail: \{jiangtao, yanguang\}@uow.edu.au).

This work was supported by Australian Research Council's DECRA Grant DE120101266, and Discovery Projects DP1093000 and DP110100736.

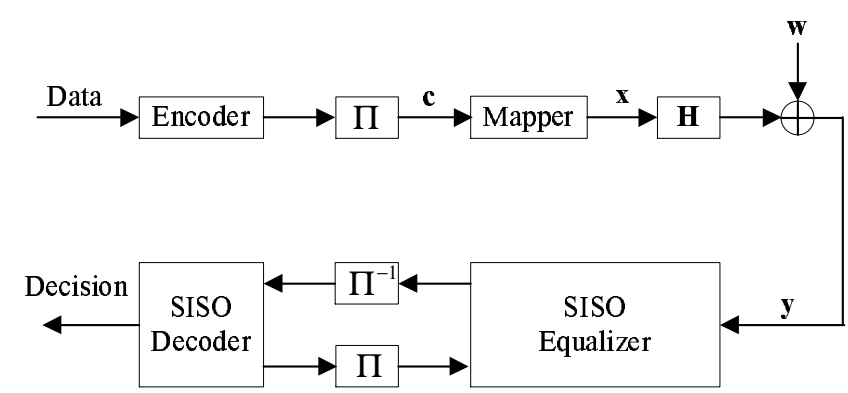

Fig. 1. A turbo equalization system, where $\Pi$ and $\Pi^{-1}$ denote the interleaver and the deinterleaver, respectively.

Recently, Donoho, Maleki and Montanari proposed the approximate message passing (AMP) technique for compressive sensing [19], [21], and it was rigorously analyzed in [20]. AMP is derived from belief propagation in which Gaussian approximations of the messages are used, and hence only means and variances are needed to approximately characterize the corresponding messages [21]. Most recently, Rangan extended AMP to generalized AMP (GAMP) for iterative estimation of a random vector whose elements are linearly mixed through a matrix and measured through a componentwise probabilistic channel [16], [17]. When the componentwise channel is the additive white Gaussian noise channel, the original AMP can be obtained from GAMP [17]. GAMP has been applied to nolinear wireless scheduling in [18], and joint sparse channel estimation and decoding in orthogonal frequency division multiplexing (OFDM) in [15].

In this work, a SISO frequency domain equalizer for singlecarrier block transmission is proposed based on GAMP. It is shown that the special structure of the system transfer matrix (which is a diagonal matrix multiplied by a discrete Fourier transfer (DFT) matrix) enables a low-complexity implementation of the GAMP algorithm. Moreover, by taking advantage of the iterative processing of a turbo receiver, iteration of the GAMP algorithm can be incorporated into the iteration between the SISO equalizer and SISO decoder. The above leads to a new SISO equalizer with complexity slightly higher than the low-complexity FD-LMMSE equalizer. However, simulation results demonstrate that a significant performance gain can be achieved by the proposed one.

The scope of this work is different from that of [15]. The work in [15] deals with joint sparse channel estimation and decoding in OFDM. The unknown vector (corresponding to $\mathbf{x}$ in [17]) estimated using GAMP is the sparse channel vector whose elements are modeled by Gaussian-mixtures, 
and the algorithm also returns the posteriors on the unknown data symbols. This work focuses on SISO equalization in single-carrier block transmission. The unknown vector (corresponding to $\mathbf{x}$ in [17]) estimated using GAMP is the data symbol vector whose elements are discrete variables with a priori distributions computed based on the feedback from the decoder.

Notations: Lower and upper case letters denote scalars. Bold lower and upper case letters represent column vectors and matrices, respectively. The superscripts $T$ and $H$ denote the transpose and conjugate transpose, respectively. $\operatorname{Diag}\left\{a_{1}, a_{2}, \ldots, a_{N}\right\}$ represents an $N \times N$ diagonal matrix with its diagonal elements give by $a_{1}, a_{2}, \ldots, a_{N}$. We use $\mathbf{I}$ to represent an identity matrix with proper size.

\section{ITERATIVE LINEAR MMSE EQUALIZATION}

The received signal for the coded single-carrier system in Fig. 1 can be represented as

$$
\mathbf{y}=\mathbf{H x}+\mathbf{w}
$$

where $\mathbf{y}$ denotes an observation vector, $\mathbf{H}$ denotes a channel matrix, w denotes a circularly symmetric Gaussian noise vector with mean $\mathbf{0}$ and covariance matrix $\sigma^{2} \mathbf{I}$, and the transmitted signal $\mathbf{x}=\left[x_{1}, x_{2}, \ldots, x_{N}\right]^{T}$ is mapped from an interleaved code sequence c, i.e., each $x_{n} \in \mathcal{A}=\left\{\alpha_{1}, \alpha_{2}, \ldots, \alpha_{2 Q}\right\}$ $\left(|A|=2^{Q}\right)$ corresponds to a length- $Q$ subsequence of $\mathbf{c}$ denoted by $\mathbf{c}_{n}=\left[c_{n}^{1}, c_{n}^{2}, \ldots, c_{n}^{Q}\right]^{T}$. Each $\alpha_{i}$ corresponds to a length- $Q$ binary sequence denoted by $\left[\alpha_{i}^{1}, \alpha_{i}^{2}, \ldots, \alpha_{i}^{Q}\right]$. We use $\mathcal{A}_{q}^{0}$ and $\mathcal{A}_{q}^{1}$ to represent the subset of all $\alpha_{i}$ with $\alpha_{i}^{q}=0$ and $\alpha_{i}^{q}=1$, respectively.

The task of the SISO equalizer is to compute the extrinsic log-likelihood ratio (LLR) for each code bit $c_{n}^{q}$ [1]-[3]

$$
L^{e}\left(c_{n}^{q}\right)=\ln \frac{P\left(c_{n}^{q}=0 \mid \mathbf{y}\right)}{P\left(c_{n}^{q}=1 \mid \mathbf{y}\right)}-L^{a}\left(c_{n}^{q}\right)
$$

where $L^{a}\left(c_{n}^{q}\right)$ is the output extrinsic LLR of the decoder in the last iteration. The extrinsic LLR $L^{e}\left(c_{n}^{q}\right)$ will be passed to the SISO decoder.

The derivation for $L^{e}\left(c_{n}^{q}\right)$ based on the linear MMSE principle can be found in [2], and the final result is shown in (16) in [2]. Recently, we presented a new derivation for $L^{e}\left(c_{n}^{q}\right)$ in [9], which is much simpler than that in [2]. Moreover, the new derivation leads to the following alternative concise representation for $L^{e}\left(c_{n}^{q}\right)$ (which turns out to be equivalent to the result in [2] as proved in [9])

$$
L^{e}\left(c_{n}^{q}\right)=\ln \frac{\sum_{\alpha_{i} \in \mathcal{A}_{q}^{0}} \exp \left(-\frac{\left|\alpha_{i}-m_{n}^{e}\right|^{2}}{v_{n}^{e}}\right) \prod_{q^{\prime} \neq q} P\left(c_{n}^{q^{\prime}}=\alpha_{i}^{q^{\prime}}\right)}{\sum_{\alpha_{i} \in \mathcal{A}_{q}^{1}} \exp \left(-\frac{\left|\alpha_{i}-m_{n}^{e}\right|^{2}}{v_{n}^{e}}\right) \prod_{q^{\prime} \neq q} P\left(c_{n}^{q^{\prime}}=\alpha_{i}^{q^{\prime}}\right)}
$$

where $m_{n}^{e}$ and $v_{n}^{e}$ are called the extrinsic mean and variance of $x_{n}$, which are defined as

$$
\begin{aligned}
v_{n}^{e} & =\left(1 / v_{n}^{p}-1 / v_{n}\right)^{-1} \\
m_{n}^{e} & =v_{n}^{e}\left(m_{n}^{p} / v_{n}^{p}-m_{n} / v_{n}\right)
\end{aligned}
$$

where $m_{n}$ and $v_{n}$ are the a priori mean and variance of $x_{n}$ calculated based on the output LLRs of the SISO decoder [2], and $m_{n}^{p}$ and $v_{n}^{p}$ are calculated by using the standard linear MMSE estimation based on model (1) [9]. The core of the linear MMSE equalizer re-derived in [9] is the standard linear MMSE estimator to compute $m_{n}^{p}$ and $v_{n}^{p}$. The computations of $m_{n}^{p}$ and $v_{n}^{p}$ can also be based on the frequency domain model (8), resulting in the FD-LMMSE equalizer.

It is worth mentioning that (3) can be obtained based on the model $m_{n}^{e}=x_{n}+\omega_{n}$ with the a priori probability of $x_{n}$ from the decoder, where $m_{n}^{e}$ represents the 'observation' and $\omega_{n}$ represents a Gaussian noise with mean 0 and variance $v_{n}^{e}$.

\section{ITERATIVE FREQUENCY DOMAIN EQUALIZATION USING GAMP}

\section{A. Frequency Domain Model}

We assume that cyclic prefixing is used in the single-carrier system, which results in a circulant channel matrix $\mathbf{H}$ in (1). But we note that, the use of cyclic prefixes is not necessary in a turbo receiver [10], [11]. A useful property of a circulant matrix is that it can be diagonalized by the Discrete Fourier Transform (DFT) matrix, i.e., $\mathbf{F H F}^{H}=\mathbf{D}$, or equivalently

$$
\mathbf{H}=\mathbf{F}^{H} \mathbf{D F}
$$

where $\mathbf{F}$ is the normalized DFT matrix (the $(m, n)$ th element is given by $N^{-1 / 2} e^{-j 2 \pi m n / N}$, where $\left.j=\sqrt{-1}\right)$, and the diagonal matrix

$$
\mathbf{D}=\operatorname{Diag}\left\{d_{1}, d_{2}, \ldots, d_{N}\right\}
$$

whose diagonal elements $\left[d_{1}, d_{2}, \ldots, d_{N}\right]^{T}=\sqrt{N} \mathbf{F h}$ ( $\mathbf{h}$ is the first column of $\mathbf{H}$ ). With (6), model (1) can be rewritten as

$$
\mathbf{z}=\mathbf{D F} \mathbf{x}+\mathbf{w}^{\prime}
$$

where $\mathbf{z}=\mathbf{F y}$, and $\mathbf{w}^{\prime}=\mathbf{F w}$ has the same distribution as $\mathbf{w}$ because $\mathbf{F}$ is a unitary matrix.

\section{B. FD-LMMSE Equalization}

To facilitate the comparison between the FD-LMMSE equalizer and the proposed GAMP based frequency domain equalizer, we summarize the FD-LMMSE equalization algorithm in the following. It is re-derived based on the frequency domain model (8) with the framework shown in Section II.

- Step 1: Compute the mean $m_{n}$ and the variance $v_{n}$ for each $x_{n}$ as follows

$$
\begin{aligned}
m_{n} & =\sum_{i=1}^{2^{Q}} \alpha_{i} P^{a}\left(x_{n}=\alpha_{i}\right) \\
v_{n} & =\sum_{i=1}^{2^{Q}}\left|\alpha_{i}-m_{n}\right|^{2} P^{a}\left(x_{n}=\alpha_{i}\right)
\end{aligned}
$$

where $P^{a}\left(x_{n}=\alpha_{i}\right)$ represents the a priori probability of $x_{n}=\alpha_{i}$, which can be calculated based on the output LLRs from the decoder.

- Step 2: Compute $m_{n}^{p}$ and $v_{n}^{p}$ based on (8) as follows

$$
\begin{aligned}
v_{1}^{p} & =v_{2}^{P}=\ldots=v_{N}^{p}=\frac{1}{N} \sum_{i=1}^{N}\left(\frac{1}{\bar{v}}+\frac{\left|d_{i}\right|^{2}}{\sigma^{2}}\right)^{-1} \\
\mathbf{m}^{p} & =\mathbf{m}+\mathbf{F}^{H} \mathbf{D}^{H}\left(\mathbf{D D}^{H}+\frac{\sigma^{2}}{\bar{v}} \mathbf{I}\right)^{-1}(\mathbf{z}-\mathbf{D F m})(12)
\end{aligned}
$$


where $\bar{v}=N^{-1} \sum_{n=1}^{N} v_{n}, \mathbf{m}=\left[m_{1}, m_{2}, \ldots, m_{N}\right]^{T}$, and $\mathbf{m}^{p}=\left[m_{1}^{p}, m_{2}^{p}, \ldots, m_{N}^{p}\right]^{T}$. Then calculate the extrinsic mean $m_{n}^{e}$ and variance $v_{n}^{e}$ for each $x_{n}$ using (4) and (5).

Once the extrinsic means and variances are available, the extrinsic LLRs for the code bits can be calculated using (3).

Here we note that, in the derivation of Step 2 of the above algorithm, we use the approximation that the a priori variances of the symbols are the same (i.e., $\bar{v}$ ). This approximation is often used in the derivation of the frequency domain equalizer such as in [10]-[12].

\section{GAMP Based Frequency Domain Equalization}

In the system represented by (1) or (8), each transmitted symbol $x_{n}(\in \mathcal{A})$ is a discrete random variable. In the linear MMSE equalization, $x_{n}$ is treated as a Gaussian variable, i.e., the equalization algorithm only utilizes the first and second order moments of $x_{n}$ (see (9) and (10) in the FD-LMMSE equalization algorithm), and it may cause performance loss.

In this work, we employ the sum-product GAMP algorithm to estimate the non-Gaussian random variables in $\mathbf{x}$. In particular, we will focus on the frequency domain model (8), i.e., the linear mixing matrix is a special matrix DF, which leads to a low complexity implementation of the GAMP algorithm.

According to the turbo principle, the output of the SISO equalizer, i.e., the input to the SISO decoder, should be extrinsic (which intuitively explains the use of extrinsic mean and variance of $x_{n}$ in (3)). Hence, we expect that the outputs of the GAMP algorithm are extrinsic to facilitate its application in turbo equalization. By examining the derivation of the original GAMP algorithm in [17], it can be found that the outputs of Step 4 in the algorithm are extrinsic as they are the messages passed from the observation side and do not contain the immediate a priori information about $x_{n}$ itself. Moreover, the output of Step 4 is in the form of mean and variance of $x_{n}$, and hence we also give them the name of extrinsic mean and extrinsic variance (but note that they are different from the extrinsic mean and variance defined in (4) and (5) in Section II). Therefore, we change the order of the steps in the original GAMP algorithm. Specifically, we make Step 5 in the original GAMP algorithm as the first step, so that Step 4 becomes the final step.

Based on the above discussion and the properties of a DFT matrix, it is not hard to obtain the following iterative algorithm to calculate the extrinsic mean and variance for each $x_{n}$.

\section{Algorithm 1}

- Initialization: Set $\mu_{r_{n}}^{-1}=0, r_{n}=0$, and $\mathbf{s}=\mathbf{0}$.

- Step 1: Compute the mean $m_{n}^{\prime}$ and the variance $v_{n}^{\prime}$ for each $x_{n}$ using the following

$$
\begin{aligned}
P_{i} & =P^{a}\left(x_{n}=\alpha_{i}\right) \exp \left(-\mu_{r_{n}}^{-1}\left|\alpha_{i}-r_{n}\right|^{2}\right) \\
P\left(x_{n}\right. & \left.=\alpha_{i}\right)=\frac{P_{i}}{\sum_{i^{\prime}=1}^{2^{Q}} P_{i^{\prime}}}, i=1,2, \ldots 2^{Q} \\
m_{n}^{\prime} & =\sum_{i=1}^{2^{Q}} \alpha_{i} P\left(x_{n}=\alpha_{i}\right) \\
v_{n}^{\prime} & =\sum_{i=1}^{2^{Q}}\left|\alpha_{i}-m_{n}^{\prime}\right|^{2} P\left(x_{n}=\alpha_{i}\right)
\end{aligned}
$$

where $P^{a}\left(x_{n}=\alpha_{i}\right)$ is the same as that in the FDLMMSE approach, i.e., it represents the a priori probability of $x_{n}=\alpha_{i}$, and can be calculated based on the output LLRs from the decoder.

- Step 2: Compute $\mu_{p_{n}}$ and $\mathbf{p}$ as follows

$$
\begin{aligned}
\mu_{p_{n}} & =\bar{v}\left|d_{n}\right|^{2} \\
\mathbf{p} & =\mathbf{D F m} \mathbf{m}^{\prime}-\Lambda_{\mathbf{p}} \mathbf{S}
\end{aligned}
$$

where $\bar{v}=N^{-1} \sum_{n=1}^{N} v_{n}^{\prime}, \mathbf{m}^{\prime}=\left[m_{1}^{\prime}, m_{2}^{\prime}, \ldots, m_{N}^{\prime}\right]^{T}$ and $\Lambda_{\mathbf{p}}=\operatorname{Diag}\left\{\mu_{p_{1}}, \mu_{p_{2}}, \ldots, \mu_{p_{N}}\right\}$.

- Step 3: Compute $\mu_{s_{n}}$ and $\mathbf{s}$

$$
\begin{aligned}
\mu_{s_{n}} & =\left(\sigma^{2}+\mu_{p_{n}}\right)^{-1} \\
\mathbf{s} & =\Lambda_{\mathbf{s}}(\mathbf{z}-\mathbf{p})
\end{aligned}
$$

where $\Lambda_{\mathbf{s}}=\operatorname{Diag}\left\{\mu_{s_{1}}, \mu_{s_{2}}, \ldots, \mu_{s_{N}}\right\}$.

- Step 4: Compute $\left\{\mu_{r_{n}}\right\}$ and $\mathbf{r}$

$$
\begin{aligned}
\mu_{r_{1}} & =\mu_{r_{2}}=\ldots=\mu_{r_{n}}=N\left[\sum_{i=1}^{N}\left(\left|d_{i}\right|^{2} \mu_{s_{i}}\right)\right]^{-1} \\
\mathbf{r} & =\mathbf{m}^{\prime}+\mu_{r_{1}} \mathbf{F}^{H} \mathbf{D}^{H} \mathbf{s}
\end{aligned}
$$

where $\mathbf{r}=\left[r_{1}, r_{2}, \ldots, r_{N}\right]^{T}$. Then return to Step 1 for the next iteration.

Compute Extrinsic LLRs: As discussed in the above, $r_{n}$ and $\mu_{r_{n}}$ are the extrinsic mean and variance of $x_{n}$. But they are not in the sense of linear MMSE, and hence they are different from those defined in (4) and (5). However, to compute the extrinsic LLRs, we can use the model $r_{n}=x_{n}+\omega_{n}^{\prime}$, where $r_{n}$ represents the 'observation', and $\omega_{n}^{\prime}$ represents a Gaussian noise with mean 0 and variance $\mu_{r_{n}}$, and therefore (3) can be used by replacing $m_{n}^{e}$ with $r_{n}$ and $v_{n}^{e}$ with $\mu_{r_{n}}$.

Equalizer Implementation: The SISO equalizer implemented with Algorithm 1 involves an inner iteration, and we give it the name Eq-GAMP-It. However, we can take advantage of the iterative processing at the turbo receiver, i.e., incorporate the iteration in Algorithm 1 into the iteration between the decoder and equalizer, thereby removing the inner iteration of the equalizer and reducing its complexity. This can be realized by setting the number of iteration in Algorithm 1 to 1 and move the initialization of Algorithm 1 into the initialization of the iterative process between the decoder and the equalizer. The resultant equalizer is called Eq-GAMP.

\section{Comparison with the FD-LMMSE Equalizer}

It can be seen that Step 1 of Algorithm 1 for the GAMP based approach functionally corresponds to Step 1 in the FDLMMSE approach, and Steps 2, 3 and 4 of Algorithm 1 for the GAMP based approach functionally correspond to Step 2 in the FD-LMMSE approach. Both approaches need to use (3) to calculate the extrinsic LLR for each code bit. Next, we compare their complexity.

Steps 2, 3 and 4 in Algorithm 1 for the GAMP based approach and their counterpart Step 2 in the FD-LMMSE approach have almost the same complexity as explained in the following. They both need one FFT (fast Fourier transform) operation and one inverse FFT operation with complexity 


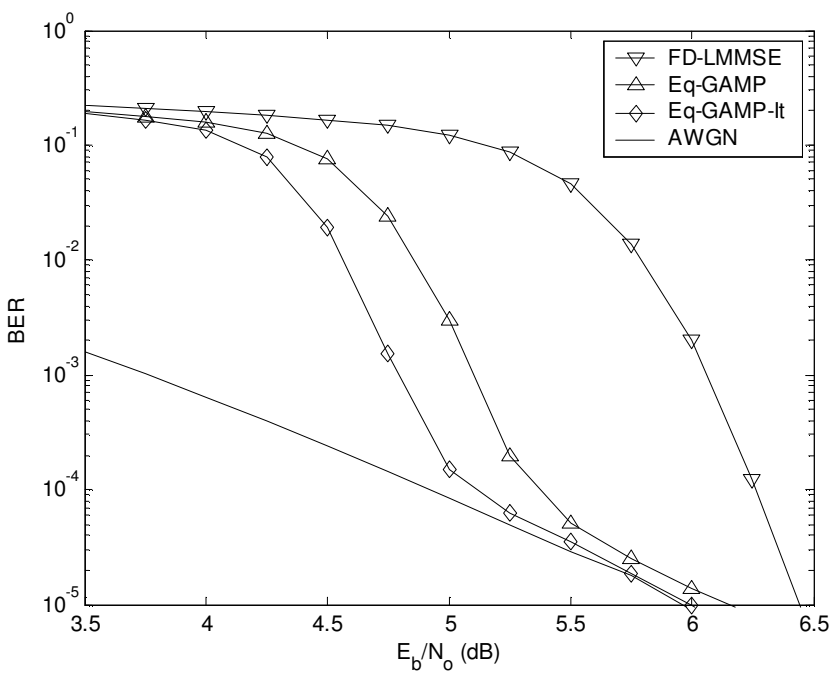

Fig. 2. BER performance of the receiver with different equalizers.

$O(\log N)$ per symbol. The remaining operations involved in Steps 2, 3, and 4 in Algorithm 1 require 14 real additions and 19 real multiplications per symbol, and the remaining operations involved in Step 2 in the FD-LMMSE approach require 15 real additions and 19 real multiplications.

The complexity difference lies in Step 1. Step 1 of Algorithm 1 for the GAMP based approach has slightly higher complexity than its counterpart Step 1 in the FD-LMMSE approach due to the extra calculations of (13) and (14) in Algorithm 1. However, thanks to (13) and (14), the symbols in the proposed approach are not simply treated as Gaussian variables. If the operation of $\exp (\cdot)$ is implemented using a look-up table approach, Step 1 in Algorithm 1 only involves marginal complexity increase.

From the above analysis, we can see that the complexity of the proposed Eq-GAMP is only slightly higher than that of the FD-LMMSE equalizer. ${ }^{1}$ However, Eq-GAMP can significantly outperform the FD-LMMSE equalizer in performance as demonstrated by the simulation results in Section IV.

\section{Simulation Results}

Consider a single-carrier block transmission system with an iterative receiver as shown in Fig. 1, where the cyclic prefixing technique is used. A rate-1/2 nonsystematic convolutional code with generator $(5,7)_{8}$ is employed, and the SISO decoder is implemented using the BCJR algorithm. The interleaved code sequence is mapped to a symbol sequence using QPSK with Gray mapping, and then the symbol sequence is divided into length-256 blocks, which are transmitted after cyclic prefixing. We use Proakis' 5-tap ISI channel with coefficients [0.227, $0.460,0.688,0.460,0.277$ ] (which generates severe distortion on the transmitted signal) to verify the performance of the approaches. The number of information bits is 7680 , and the number of iterations is 10 .

The performance of the turbo receiver with the proposed Eq-GAMP and the FD-LMMSE equalizer is compared in
Fig. 2, where the performance of the code over AWGN channel (which is the performance limit of the system over ISI channels) is also shown. It can be seen that a significant performance gain is achieved by Eq-GAMP compared with the FD-LMMSE equalizer. The performance of the receiver with Eq-GAMP-It (5 iterations in Algorithm 1) is also shown for reference. Eq-GAMP-It slightly outperforms Eq-GAMP at the cost of considerable complexity increase.

\section{REFERENCES}

[1] C. Douillard, M. Jezequel, C. berrou, A. Picart, P. Didier, and A. Glarieux, "Iterative correction of intersymbol interference: Turbo-equalization," Eur. Trans. Telecommun., vol. 6, pp. 507-511, Sept./Oct. 1995.

[2] M. Tüchler, A. C. Singer,and R. Koetter, "Minimum mean squared error equalization using a priori information," IEEE Trans. Signal Process., vol. 50, no. 3, pp. 673-683, Mar. 2002.

[3] R. Koetter, A. C. Singer, and M. Tüchler,"Turbo equalization,” IEEE Signal Process. Mag., vol. 21, pp.67-80, Jan. 2004.

[4] C. Laot, A. Glavieux, and J. Labat, "Turbo equalization: Adaptive equalization and channel decoding jointly optimized," IEEE J. Sel. Areas Commun., vol. 19, no. 9, pp. 1744-1752, Sep. 2001.

[5] X. Wang and H. V. Poor, "Iterative (turbo) soft interference cancellation and decoding for coded CDMA," IEEE Trans. Commun., vol. 47, pp.1046-1041, Jul. 1999.

[6] M. Tüchler, R. Koetter, and A. C. Singer, "Turbo equalization: principles and new results," IEEE Trans. Commun., vol. 50, no. 5, pp. 754-767, May 2002.

[7] Q. Guo and Li Ping,"LMMSE turbo equalization based on factor graphs," IEEE J. Sel. Areas Commun., vol. 26, no. 2. pp. 311-319, Feb. 2008.

[8] M. Tüchler and J. Hagenauer, "Turbo equalization using frquency domain equalizers," in Proc. Allerton Conference, Monticello, IL, USA, Oct. 2000, pp. 1234-1243.

[9] Q. Guo and D. Huang, "A concise representation for the soft-in soft-out LMMSE detector," IEEE Commun. Lett., vol. 15, pp. 566-568, May 2011.

[10] Q. Guo, Li Ping, and D. Huang, "A Low-complexity iterative channel estimation and detection technique for doubly selective channels," IEEE Trans. Wireless Commun., vol. 8, pp. 4340-4349, Aug. 2009.

[11] H. Liu and P. Schniter, "Iterative frequency-domain channel estimation and equalization for single-carrier transmissions without cyclic-prefix," IEEE Trans. Wireless Commun., vol. 7 no. 10, pp. 3686-3691, Oct. 2008.

[12] A. Gusmão, P. Torres, R. Dinis, and N. Esteves, "A turbo FDE technique for reduced-CP SC-based block transmission systems," IEEE Trans. Commun., vol. 55, no. 1, pp. 16-20, Jan. 2007.

[13] D. Falconer, S. L. Ariyavisitakul, A. Benyamin-Seeyar, and B. Eidson, "Frequency domain equalization for single-carrier broadband wireless systems," IEEE Commun. Mag., vol. 40, pp. 58-66, apr. 2002.

[14] M. Stojanovic, "Underwater acoustic communications: design considerations on the physical layer," in Proc. IEEE/IFIP WONS 2008, Germany, pp. 1-10, Jan. 2008.

[15] P. Schniter, "A message-passing receiver for BICM-OFDM over unknown clustered-sparse channels," IEEE J. Sel.Topics in Signal Process., vol. 5, no. 8, pp. 1462-1474, Dec. 2011.

[16] S. Rangan, "Generalized approximate message passing for estimation with random linear mixing," in Proc. Int symp. Inf. Theory, Aug. 2011, pp. $2168-2172$.

[17] S. Rangan, "Generalized approximate message passing for estimation with random linear mixing,"arXiv:1010.5141v1 [cs.IT], Oct 2010.

[18] S. Rangan and R. K. Madan,"Belief propagation methods for intercell interference coordination in femtocell networks," IEEE J. Sel. Topics Signal Process., pp. 631-640, Apr. 2012.

[19] D. L. Donoho, A. Maleki, and A. Montanari,"Message-passing algorithms for compressed sensing," Proc. Nat. Acad. Sci., vol. 106, no. 45, pp. 18914-18919, Nov. 2009.

[20] M. Bayati and A. Montanari,"The dynamics of message passing on dense graphs, with applications to compressed sensing," IEEE Trans. Inform. Theory, vol. 57, no. 2, pp. 764-785, Feb. 2011.

[21] D. L. Donoho, A. Maleki, and A. Montanari,"Message-passing algorithms for compressed sensing: I. motivation and construction," in Proc. IEEE Inform. Theory Workshop, Jan. 2010.

${ }^{1}$ Eq-GAMP-It may have significantly higher complexity than the FDLMMSE equalizer, depending on the number of iterations in Algorithm 1. 